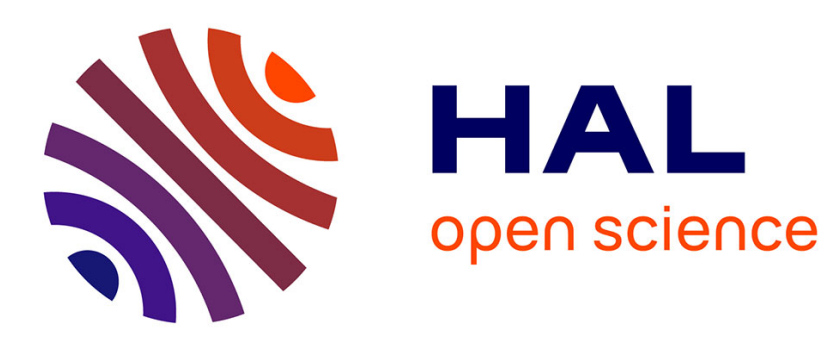

\title{
Conditions for detection of ground deformation induced by conduit flow and evolution
}

F. Albino, Virginie Pinel, H. Massol, M. Collombet

\section{To cite this version:}

F. Albino, Virginie Pinel, H. Massol, M. Collombet. Conditions for detection of ground deformation induced by conduit flow and evolution. Journal of Geophysical Research: Solid Earth, 2011, 116, pp.B06201. 10.1029/2010jb007871 . ird-00607732

\section{HAL Id: ird-00607732 \\ https://hal.ird.fr/ird-00607732}

Submitted on 11 Jul 2011

HAL is a multi-disciplinary open access archive for the deposit and dissemination of scientific research documents, whether they are published or not. The documents may come from teaching and research institutions in France or abroad, or from public or private research centers.
L'archive ouverte pluridisciplinaire HAL, est destinée au dépôt et à la diffusion de documents scientifiques de niveau recherche, publiés ou non, émanant des établissements d'enseignement et de recherche français ou étrangers, des laboratoires publics ou privés. 


\section{Conditions for detection of ground deformation induced by conduit flow and evolution}

F. Albino ${ }^{1}$, V. Pinel ${ }^{1}$, H. Massol ${ }^{2}$ and M. Collombet ${ }^{1}$

F. Albino, ISTerre, CNRS, IRD, Université de Savoie, Campus scientifique, 73376 Le Bourget du Lac Cedex, FRANCE. (Fabien.Albino@univ-savoie.fr)

M. Collombet, ISTerre, CNRS, IRD, Université de Savoie, Campus scientifique, 73376 Le Bourget du Lac Cedex, FRANCE.

H. Massol, Laboratoire IDES, Département des Sciences de la Terre, Université Paris XI, 91405 Orsay Cedex, FRANCE.

V. Pinel, ISTerre, CNRS, IRD, Université de Savoie, Campus scientifique, 73376 Le Bourget du Lac Cedex, FRANCE.

${ }^{1}$ ISTerre, CNRS, IRD, Université de

Savoie, Campus scientifique, 73376 Le

Bourget du Lac Cedex, FRANCE.

${ }^{2}$ Lab. IDES, Département des Sciences de

la Terre, Université Paris XI, 91405 Orsay

Cedex, FRANCE. 
Abstract. At mature andesitic volcanoes, magma can reach the surface through the same path for several eruptions thus forming a volcanic conduit. Due to degassing, cooling and crystallization, magma viscosity increase in the upper part of the conduit may induce the formation of a viscous plug. We conducted numerical simulations to quantify the deformation field caused by this plug emplacement and evolution. Stress continuity between Newtonian magma flow and elastic crust is considered. Plug emplacement causes a ground inflation correlated to a decrease of the magma discharge rate. A parametric study shows that surface displacements depend on three dimensionless numbers: the conduit aspect ratio (radius/length), the length ratio between the plug and the conduit and the viscosity contrast between the plug and the magma column. Larger displacements are obtained for high viscosity plugs emplaced in large aspect ratio conduits. We find that only tiltmeters or GPS located close the vent (a few hundred meters) might record the plug emplacement. At immediate proximity of the vent, plug emplacement might even dominate the deformation signal, over dome growth or magma reservoir pressurization effects. For given plug thicknesses and viscosity profiles, our model explain well the amplitude of tilt variations (from 1 to $25 \mu \mathrm{rad}$ ) measured at Montserrat and Mt. St. Helens. We also demonstrate that, at Montserrat, even if most of the tilt signal is due to shear stress induced by magma flow, pressurization beneath the plug account for 20 percent of the signal. 


\section{Introduction}

1 Cyclic patterns of activity, with a succession of episodes of lava dome growth and 2 quiescent periods, have been observed at many andesitic volcanoes, such as Soufrière Hills ${ }_{3}$ (Montserrat, 1995-present), Mt St Helens (USA, 1980-1986), Merapi (Philippines, 1768${ }_{4}$ 1998), Unzen (Japan, 1990-1995) or Santiaguito (Guatemala, 1922-2000) [Voight et al., ${ }_{5}$ 1999; Swanson and Holcomb, 1990; Voight et al., 2000; Nakada et al., 1999; Harris et al., 6 2002]. Large fluctuations in discharge rate are correlated with fluctuations of various 7 signals such as ground deformation, seismicity and gas emissions. For long-term cycles, 8 (months to years), the mechanism invoked is pressure changes occurring within a magma 9 reservoir [Melnik and Sparks, 2005]. In some volcanic areas such as Soufrière Hills, Mt St

Helens or Merapi, improvement of volcanic monitoring, with acquisitions of high frequency and precise data, has permitted to identify cyclic patterns with a period of only a few hours, during phases of dome extrusion [Voight et al., 1999]. Whereas cyclic deformation at long time-scales is attributed to magma reservoir dynamics, short time-scale variations are explained by the non-linear dynamics of magma flow in the volcanic conduit [Denlinger and Hoblitt, 1999; Voight et al., 1999; Wylie et al., 1999; Lensky et al., 2008; Collier and Neuberg, 2006]. Intermediate cycles with periods of several weeks have been attributed to the capacitor effect of dykes connecting magma reservoirs and upper conduits [Costa et al., 2007b]. Also, sudden events characteristic of andesitic volcanic activity, such as dome collapse, can induce rapid pressure changes within the magma reservoir [Voight et al., 2006]. It follows that a combination of changes taking place at different levels of the magma plumbing system, causes the temporal evolution of volcanic activity. In order 
$\mathrm{X}-4$ ALBINO ET AL.: GROUND DEFORMATION INDUCED BY CONDUIT FLOW

22

to improve eruption forecasting, it is required to study magma flow conditions in the volcanic conduit of andesitic volcanoes and how it affects the monitoring signals recorded at the surface.

Previous work [Sparks, 1997; Melnik and Sparks, 1999; Barmin et al., 2002], focussing on changes of the physical properties of the ascending magma, has shown that gas loss and crystallization occur mainly within the last hundred of meters below the surface. Both mechanisms may induce a strong increase in magma viscosity of several orders of magnitude [Shaw, 1963; Hess and Dingwell, 1996; Llewellin and Manga, 2005]. For example, the viscosity of an andesitic magma at $900{ }^{\circ} \mathrm{C}$ containing $5 \mathrm{wt} \%$ is around $10^{4}$ Pa.s, but can reach values of $10^{11}$ Pa.s if the magma is fully degassed [Sparks, 1997]. In this process, vertical as well as lateral gas escape may have a strong impact on the resulting viscosity profiles [Collombet, 2009]. It has been shown that degassing may be coupled with crystallization which, in turn, also affects magma viscosity [Sparks, 1997]. Costa et al. [2007a] consider a strong dependency of the viscosity on crystal content, with an increase in crystal content from 50 to 80 vol \% leading to an increase in viscosity by a factor of $10^{8}$. As a consequence of these shallow crystallization and degassing processes, a more viscous body, so-called plug, is typically emplaced at the upper part of the conduit. The presence of this degassed portion in the upper part of the conduit has been confirmed by petrological studies performed on explosive deposits at Soufrière Hills Volcano, Montserrat [Burgisser et al., 2010]. The plug formation tends to reduce the magma flow rate and also induces a pressurization within the conduit. Changes in magma rheology modify the overall flow dynamics within the conduit and, consequently, the stress field within the surrounding crust, which might therefore induce precursor signals such as deformation 
45

or seismicity. Ground deformation related to conduit flow processes has been studied through analytic solutions [Bonaccorso and Davis, 1999; Nishimura, 2006, 2009] as well as numerical modelling [Chadwick et al., 1988; Beauducel et al., 2000; Green et al., 2006; Hautmann et al., 2009]. However, deformation of the conduit wall due to the magma flow was not taken into consideration and most of studies considered only one component of the stress field acting on the wall rocks, either shear stress [Beauducel et al., 2000; Green et al., 2006] or normal stress [Hautmann et al., 2009]. Only few studies consider both components [Chadwick et al., 1988; Anderson et al., 2010]. However, in these studies, the two effects are evaluated independently despite their obvious link through flow motion equations [Nishimura, 2009].

Here, we consider the full coupling between the fluid flow and crustal deformation, taking into account the total stress field and the deformation of the conduit wall. We carry out numerical calculations in axial geometry to model the displacement field induced by a steady flow when a plug is emplaced at the top of the conduit. We perform a parametric study in order to quantify the deformation field induced by the increase of magma viscosity at the upper part of the conduit and give an estimation of the distance of detection of the induced signal. We then compare the magma flow rate and the surface tilt expected during plug growth within the conduit. We further discuss our results with regards to data recorded at the two most studied andesitic volcanoes, Soufrière Hills (Montserrat) and Mount St Helens (USA), and consider more realistic flow conditions as well as data acquisition geometry (including volcano topography). 
$X-6$

ALBINO ET AL.: GROUND DEFORMATION INDUCED BY CONDUIT FLOW

\section{Model}

\subsection{Description}

${ }_{66}$ Magma flow through a volcanic conduit embedded in the crust is modelled using a

${ }_{67}$ "Finite Element Method" (FEM) in axisymmetrical geometry (COMSOL software). The ${ }_{68}$ conduit is a vertical cylinder with radius $\left(a_{c}\right)$ and length $\left(L_{c}\right)$. The magma, considered as

${ }_{69}$ a Newtonian fluid, flows with steady state conditions due to an overpressure $\left(P_{c}\right)$, which

${ }_{70}$ is an excess of pressure comparing to the lithostatic state, applied at the conduit bottom.

${ }_{71}$ This excess pressure is due to the presence of an overpressurized storage zone, which effect

72 is not modeled in the present study. Our model is not time dependent and describes the departure induced by a given steady state magma flow within the conduit, from a

reference state being a lithostatic state of stress within the surrounding rocks with an overpressurized reservoir not connected to the surface. It means that all the displacement and state of stress calculated are the one induced by the magma flow within the conduit. The surrounding crust is treated as a homogeneous elastic medium, characterized by its shear modulus $(G)$ which will be variable and its Poisson's ratio $(\nu)$ which is fixed at 0.25 in all our study. Boundary conditions used for the host rock medium are the following (see Figure 1): (i) free displacements at the top boundary, which corresponds to the ground surface ; (ii) no vertical displacement at the bottom boundary and (iii) no radial displacement at the external lateral boundary. In order to neglect the boundary effects, the external lateral boundary of the elastic medium is located far away from the conduit (at a distance of $100 \mathrm{~km}$ ). A full coupling of magma flow and crustal deformation is considered by applying the continuity of the stress field (Fig. 1), including normal as well as tangential components, at the conduit wall. Conduit wall deformation is calculated 
87

by an iterative process as described in Appendix A. Geometry, physical properties and boundary conditions applied in our model are shown in Figure 1a.

The steady state of fluid flow is solved from the Navier \& Stokes equation. A complete parametric study is performed for an incompressible fluid with a constant density $\left(\rho_{m}\right)$ equal to the surrounding crustal density, a viscosity function $(\mu(z))$ and characterized by a Poiseuille flow. We later consider in the discussion a compressible fluid associated to complex and realistic flow conditions. In the incompressible case, the component of the stress normal to the conduit wall is equal to the magma pressure. We first calculate perturbations of the reference state induced by the flow of a constant viscosity magma. This solution is then considered as our new state of reference. We then quantify displacements induced by the emplacement of a more viscous portion of the flow, so-called plug, at the top of the conduit with respect to the constant viscosity case.

To first order, the increase of viscosity is approximated by a step function (Fig. 1b) that is dependent on the conduit depth. The plug is characterized by two parameters: its viscosity $\left(\mu_{p}\right)$, with $\mu_{p}>\mu_{m}$ ( $\mu_{m}$ being the viscosity of the magma column) and its length $\left(h_{p}\right)$, with $h_{p}<\frac{1}{2} L_{c}$. For Poiseuille flow, with a constant viscosity, expressions for the stress field components at the conduit walls are simplified: (i) the normal stress is equivalent to the fluid pressure and varies linearly with depth, $\frac{d P}{d z}=$ cste; (ii) the tangential stress or shear stress is a constant value, which only depends on the pressure gradient and the conduit radius, $\tau=\left(\frac{a_{c}}{2}\right)\left(\frac{d P}{d z}\right)=$ cste. In that case, and providing that the conduit deformation remains small (see Appendix A for conditions), we solve equations for the stress and displacement field within the elastic medium, applying the stress components corresponding to the fluid flow at the conduit wall. For magmas with 
constant viscosity (our reference case), the pressure gradient as well as the tangential stress applied at the conduit wall are constant $\tau_{r e f}=\frac{a_{c} P_{c}}{2 L_{c}}$ (the dashed curve on Fig. 1b). In this case, for a given Poisson's ratio, the surface displacements are a function of the conduit geometry $\left(a_{c}, L_{c}\right)$, the overpressure at the bottom of the conduit $\left(P_{c}\right)$ and the shear modulus $(G)$ only. They do not depend on the viscosity value. For a plug model, the tangential stress is different from the reference case both at the upper part of the conduit $\left(\tau_{\text {up }}\right)$ and at the lower portion $\left(\tau_{\text {low }}\right)$ (solid curve in Fig. 1b). The increase of magma viscosity within the upper part induces an overpressure when compared to the reference case (of constant viscosity). This overpressure reaches a maximum value, denoted $\Delta P_{p}$ at the base of the plug (Fig. 1b). At the plug bottom, the magma pressure, $P_{p}$, can be derived using the conservation of the volumetric flux along the conduit:

$$
P_{p}=\frac{\mu_{p} h_{p}}{\mu_{p} h_{p}+\mu_{m}\left(L_{c}-h_{p}\right)} P_{c}
$$

where $P_{c}$ is the excess pressure at the conduit bottom. It follows that the overpressure $\left(\Delta P_{p}\right)$ can be expressed as a function of the viscosity contrast and the length ratio:

$$
\Delta P_{p}=P_{p}-\frac{h_{p}}{L_{c}} P_{c}=\left(\frac{\left(\frac{\mu_{p}}{\mu_{m}}\right)\left(\frac{h_{p}}{L_{c}}\right)}{1+\frac{h_{p}}{L_{c}}\left(\frac{\mu_{p}}{\mu_{m}}-1\right)}-\frac{h_{p}}{L_{c}}\right) P_{c}
$$

This overpressure is equal to zero when there is no viscosity contrast, $\mu_{p}=\mu_{m}$, and reaches the value $P_{c}\left(1-\frac{h_{p}}{L_{c}}\right)$ when the viscosity within the plug tends to infinity. For the plug case, the two magma viscosities, $\mu_{m}$ and $\mu_{p}$, as well as the length of the plug, $h_{p}$, have an effect on the displacement. For a given Poisson's ratio and using the conduit length $\left(L_{c}\right)$ as a length scale and the term $\left(\frac{P_{c}}{G} L_{c}\right)$ as a displacement scale, dimensionless solutions are only dependent on the following three dimensionless numbers: (i) the conduit aspect ratio 
${ }_{131}\left(\frac{a_{c}}{L_{c}}\right)$, (ii) the length ratio between the plug and the total conduit $\left(\frac{h_{p}}{L_{c}}\right)$, (iii) the viscosity 132 ratio between the two magmas $\left(\frac{\mu_{p}}{\mu_{m}}\right)$.

\subsection{Main Limitations}


before extrusive activity. One of the consequence is that the cylinder conduit is probably limited to the last hundred meters, but it can reach several kilometers in case of high fragmentation and large depressurization. The extension at depth of this cylindrical shape is still under debate. Some authors suggest a possible connection between a shallow cylindrical conduit and a deep dyke [Costa et al., 2007b, a]. The effect of the pressurized dyke as well as a pressurized magma reservoir below the cylindrical conduit will act on magma flux and also on ground deformation on large time scale, over weeks to year. Here, we are interested in the short time scale deformation only occurring during rapid changes in magma flow dynamics at shallow level. In consequence, our model only focus on the shallow part of a mature magmatic system associated to cylindrical shapes, which has already been considered in many studies in relation with conduit flow [Wylie et al., 1999; Melnik and Sparks, 1999; Barmin et al., 2002; Collier and Neuberg, 2006; Lensky et al., 2008; Collombet, 2009].

The second main assumption of our model is the choice of a Newtonian behaviour for the magma. Indeed, different processes, such as magma crystallization and cooling or high volatile contents may lead to a non-Newtonian behaviour, implying a dependence of the viscosity with the shear rate. The non-Newtonian behaviour is reached for high crystalbearing magmas, containing more than 30-40\% of crystals [Pinkerton and Stevenson, 1992; Lejeune and Richet, 1995]. This threshold can be reached in the case of Soufrière Hills [Sparks et al., 2000] or Mt. St Helens [Gardner et al., 1996] volcanoes if the andesitic magmas spend a significant duration in the conduit. Recent publications, such as [Caricchi et al., 2007] show that, the magma behaviour is also highly dependent on the strain rate considered and for high values (above $10^{-4} \mathrm{~s}^{-1}$ ) magma overall viscosity decreases with 
strain rates exhibiting non-Newtonian behaviour. In that case, the velocity profile should departs from the Poiseuille parabolic profile affecting values of the shear stress at the conduit wall. Considering Figure 3 from Caricchi et al. [2007] we can predict that the shear stress may decrease of approximately one order of magnitude comparing with the Newtonian case.

Another strong limitation of our model comes from the fact that thermal effects are not taken into account. However they could potentially favor non-Newtonian behaviour of the magma as well as affect stresses applied at the conduit wall. During ascent of hot magma in a cold crust, large thermal gradient can develop at the conduit wall. Mechanism of magma cooling or host rocks heating due to viscous effect can occur and the dominant effect depends on the flow conditions [Costa et al., 2007c]. For example, a thermal boundary layer due to cooling effect should represent less than $0.5 \mathrm{~m}$ of the conduit radius and leads to a decrease of 100 to $200 \mathrm{~K}$ of temperature [Collier and Neuberg, 2006]. In opposite way, viscous heating effects can produce an increase of temperature at the wall and induce changes in the velocity profile of the flow, which evolves from parabolic (as we consider in our paper) to plug-like [Costa and Macedonio, 2005; Costa et al., 2007c]. But these variations in temperature near the wall implies a melt viscosity change of one order of magnitude [Hess and Dingwell, 1996], which is much less than viscosity changes due to gas loss during magma ascent [Sparks, 1997]. In consequence, we believe that, despite this simplification, we capture the first order effect in our model. 


\section{Results}

\subsection{Surface Displacements}

In this section, we explore a range of parameters for each dimensionless number to describe the influence of various parameters on the ground deformation. In each model, radial $\left(U_{r}\right)$, vertical $\left(U_{z}\right)$ displacements and tilt $\left(\frac{\delta U_{z}}{\delta r}\right)$ at the surface are calculated. Radial and vertical displacements are positive when directed, respectively, outward and up from the conduit wall (see r,z direction in Fig. 1a). Tilts are negative when the surface is moving upward going toward the vent, which corresponds to a ground inflation. For all calculations, the Poisson's ratio value is 0.25 . Results for the reference state (i.e. constant viscosity: $\left.\frac{\mu_{p}}{\mu_{m}}=1\right)$ for three different conduit aspect ratios $\left(\frac{a_{c}}{L_{c}}\right)$ are shown in Figure 3 . The displacement trend is similar for various conduit aspect ratios except in the vicinity of the vent where differences are observed for radial distances less than $20 \%$ of the conduit length. The amplitude of the displacements and tilts is more important for larger values of $\frac{a_{c}}{L_{c}}$, corresponding to the largest or shortest conduits. This means, as previously shown by Chadwick et al. [1988], that a larger pressure is required to explain a given displacement when considering a smaller conduit radius. In Figure 3, we also show that displacements are both induced by the shear stress component and by the pressure. Both components induce displacements of the same order of amplitude. For radial displacements, shear stress has a strong influence in the near field (for $\frac{r}{L_{c}}<0.5$ ) whereas pressure has more effect in the far field (for $\frac{r}{L_{c}}>0.5$ ). For vertical displacements and tilts, pressure and shear stress induce opposite ground movement. Shear stress induces inflation whereas pressure induces deflation. However, the amplitude of the shear stress effect is larger, which results in a total displacement directed upward. In this case, neglecting the pressure component 
leads to an overestimate in the induced displacement field. For near field measurements, this overestimation is larger for a smaller conduit aspect ratio $\left(\frac{a_{c}}{L_{c}}\right)$. For example, our model gives an overestimation, larger than $20 \%$ for the tilt at a distance of $500 \mathrm{~m}$ from the conduit, if pressure at the conduit wall is neglected, with the conditions taken by Green et al. [2006] to model tilt recorded at Montserrat volcano (i.e.: a shear stress of $0.5 \mathrm{MPa}$ applied on the wall of a conduit with a $1000 \mathrm{~m}$ length and a $15 \mathrm{~m}$ radius and a Young's modulus fixed at $2 \mathrm{GPa}$ ). Thus, each stress component has an important effect on the total displacement field and none of them should be neglected in deformation models.

In case a viscous plug is present at the top of the conduit, displacements larger than those described above, for the constant viscosity case, are induced. We calculate surface tilts due to plug flow models exploring the range of $10^{0}-10^{5}$ for the viscosity ratio $\left(\frac{\mu_{p}}{\mu_{m}}\right)$ and 0-0.5 for the length ratio $\left(\frac{h_{p}}{L_{c}}\right)$. Figure 4 shows the tilts calculated at a radial distance of $500 \mathrm{~m}$ from the conduit, for the tested parameter range, considering a conduit of $15 \mathrm{~m}$ radius and $5000 \mathrm{~m}$ length. Magma pressure at the conduit bottom $\left(P_{c}\right)$ and the elastic shear modulus $(G)$ of the crust are respectively fixed at $10 \mathrm{MPa}$ and $0.8 \mathrm{GPa}$. Such values seem realistic for Soufrière Hills [Green et al., 2006; Costa et al., 2007b; Voight et al., 1999]. Tilt calculation is relative to our reference case obtained with constant viscosity, such that it's value tends to zero when either $h_{p}$ tends to zero or $\frac{\mu_{p}}{\mu_{m}}$ tends to 1. Figure 4a shows that the plug emplacement induces an inflation (negative tilt values). At $500 \mathrm{~m}$ from the conduit wall, tilt values reach more than $3 \mu \mathrm{rad}$ for thin plugs characterized by a large viscosity contrast $\left(\frac{\mu_{p}}{\mu_{m}}>10^{2.5}\right.$ and $\left.\frac{h_{p}}{L_{c}}<0.05\right)$. However the largest displacements are not always obtained with the thinnest plugs: for small viscosity ratios $\left(\frac{\mu_{p}}{\mu_{m}}<10^{3}\right)$, there exists a critical plug thickness, $h_{p(c r i t)}$, corresponding to a maximum of the induced tilt 
amplitude. For large viscosity ratios $\left(\frac{\mu_{p}}{\mu_{m}}>10^{3}\right), h_{p(c r i t)}$ tends to zero: the tilt increases when the plug length decreases. In addition, for large viscosity ratio, tilt does not depend on the viscosity ratio, but is mainly a function of the plug length. The influence of each stress component on the ground displacements is also shown in Figure 4b and 4c. Even if the pressure acting along the conduit wall does not dominate the tilt signal, it has a strong influence inducing a ground deflation (positive tilt values). The effect is maximum, with an amplitude larger than $1 \mu \mathrm{rad}$, for intermediate length ratios (between 0.1 and 0.3). In most cases, except for the thinnest plugs, neglecting the pressure effect leads to overestimate the induced tilt, which means that the tangential stress component will be underestimated when interpreting an observed signal.

\subsection{Detection of Ground Deformation}

We have showed that an increase of magma viscosity occurring at the top of a conduit, increases tilts at the ground surface. In order to guide the choice for in-situ instrument types and locations, it seems important to quantify the maximal distance from the volcanic vent, where this signal can be detected. We estimate this critical distance, hereafter called "detection distance" using a threshold value of detection for each component of the displacement. Horizontal and vertical ground movements can be detected by GPS receivers. The theoretical precision of the instrumentation used in volcanology is around $5 \mathrm{~mm}$ and $10 \mathrm{~mm}$ respectively for the horizontal and vertical displacement (http://www.igage.com/GPSaccy/index.html). However, field measurements can be perturbed by the atmospheric component or local perturbations of the instrumented site. We thus assume in our study that only millimetric changes for the radial displacements and centimetric changes for the vertical displacements will be detected. On the other hand, 
a more accurate method to detect ground motion is to measure the tilt of the volcano surface. According to the tiltmeters used, ground deformation less than 1 rrad can be detected (http://www.carboceramics.com/Tiltmeters-Clinometers), one microradian corresponding to a vertical variation of one millimeter over a distance of one kilometer. This type of measurement is difficult to set up because instruments are highly sensitive to changes in temperature or atmospheric pressure. In order to minimize the influence of these external effects on recorded measurements, tiltmeters are often placed in boreholes, several centimeters beneath the surface. Here, we choose an upper value for our detection threshold and we consider that only tilt variations larger than $1 \mu \mathrm{rad}$ are detected.

We use the same model parameters as in Figure $4\left(G=0.8 \mathrm{GPa}, \nu=0.25, P_{c}=10\right.$ $\mathrm{MPa}, L_{c}=5000 \mathrm{~m}$ and $a_{c}=15 \mathrm{~m}$ ) and threshold values of $1 \mathrm{~mm}$ and $1 \mathrm{~cm}$ respectively for the radial and the vertical displacements, and $1 \mu \mathrm{rad}$ for the tilt. We find that: (i) radial displacement is never detectable for plugs representing more than $20 \%$ of the total conduit, but it can be observed over $1 \mathrm{~km}$ for plugs with length ratio less than $10 \%$ and viscosity contrast more than $10^{2}$ (see figure 5a). (ii) vertical displacement can never be detected, except a few meters from the conduit for the thinnest plugs (see figure 5b). (iii) tilt can be detected for a few hundred meters depending on the plug characteristics as detailed in Figure 6c. Tiltmeters or GPS located at a few hundred meters from the conduit are thus appropriate to detect the ground deformation, respectively tilt or radial displacement, induced by plug emplacement within the upper part of a volcanic conduit. As previously explained, the amplitude of the displacements is not only dependent on the viscosity ratio $\left(\frac{\mu_{p}}{\mu_{m}}\right)$ and length ratio $\left(\frac{h_{p}}{L_{c}}\right)$ but is also function of the balance between the reservoir overpressure and the host rocks elastic properties, $\frac{P_{c}}{G}$, as well as the conduit 
aspect ratio $\left(\frac{a_{c}}{L_{c}}\right)$. Figure 6 shows the effect of these two parameters on the detection distance. The case $\mathrm{c}$ ) is calculated for a radius $a_{c}$ equal to $15 \mathrm{~m}$ and a shear modulus $G$ equal to $0.8 \mathrm{GPa}$, values previously used. The case a) provides the detection distance for a larger radius $\left(a_{c}\right)$, the case d) for a larger shear modulus $(G)$ and the case b) when both parameters are increased. As expected, an increase of the conduit radius or a decrease of the host rocks shear modulus, tends to increase the detection distance for tilt signal. During the extrusive phase, the detection of ground deformation caused by plug emplacement will be much easier in the case of andesitic volcanoes with a large conduit embedded in soft host rocks.

\subsection{Flow Rate Versus Tilt Signal During Plug Evolution}

The formation of a viscous plug at the top of the volcanic conduit induces a decrease of magma flow. The flow rate for a plug model can be expressed as a function of the reference flow rate, $Q_{\text {ref }}$, of the "constant viscosity" case:

$$
Q=\left(\frac{1}{1+\frac{h_{p}}{L_{c}}\left(\frac{\mu_{p}}{\mu_{m}}-1\right)}\right) Q_{r e f}
$$

In the previous section, we showed that plug emplacement also induces ground motion signals large enough to be detected under certain conditions. During the extrusive phases of andesitic volcanoes, a plug may form in the upper portion of the conduit and evolve through time due to continuous degassing as well as cooling and crystallization processes. Plug evolution can proceed either through an increase of its viscosity or its size. In nature, both cases are probably mixed, but here we choose to compare the relative evolution of tilt and flow rates observed at the surface as a consequence of these two end-members (Figure 7). 
For a given plug length, an increase of plug viscosity reduces, as expected, the magma

flow rate produced at the surface (Fig. 7b, left panel). As a consequence of this flow rate decrease, the amount of time required to entirely extrudes the plug considered (thickness $h_{p}$ and viscosity $\mu_{p}$ ), increases (Fig. 7c, left panel). This duration corresponds to the expected duration of the deflation induced by the plug extrusion. Whatever the value of $\frac{h_{p}}{L_{c}}$, the flow rate falls below $5 \%$ of its reference value for a viscosity contrast larger than $10^{3}$. This increase of viscosity induces a ground inflation: the tilt amplitude first increases with the viscosity contrast before reaching a constant and maximum value (Fig. 7a, left panel). The threshold value of viscosity at which the maximum of tilt amplitude is reached, depends on the plug size, with larger values for thinner plugs. For plugs that are more viscous than the threshold value, the tilt amplitude remains constant and the magma flow rate is close to zero. Thus, if a high contrast of viscosity already exists between the plug and the magma column, the evolution of the plug viscosity can not be detected from the surface displacement measurements.

The other end-member behaviour is the one induced by a thickening plug of constant viscosity. Once again due to plug evolution, the magma flow rate decreases (Fig. 7b, right panel) and the extrusion duration consequently increases (Fig. 7c, right panel). However for small viscosity contrasts, the flow rate reduction remains small. For the induced tilt (Fig. 7a, right panel), the behaviour is different than in the previous case: we observe a rapid ground inflation (upward displacement when going towards the crater), until the plug reaches its critical thickness, $h_{p(c r i t)}$, as previously defined. Once the plug thickness is larger than this critical value, the tilt amplitude decreases together with the flow rate. For a large viscosity contrast, the critical thickness tends to zero, and the first phase can 
comparison of the relative amplitude of these various phenomena. It shows displacements induced by (i) surface pressure change of $1 \mathrm{MPa}$ related to the emplacement/removal of a lava dome (1 MPa corresponding to a height change of $60 \mathrm{~m}$ of a material with a density of $1700 \mathrm{~kg} \cdot \mathrm{m}^{-3}$ ), (ii) an overpressure variation of $1 \mathrm{MPa}$ occurring within a magma reservoir and (iii) the emplacement of a plug in the upper part of the conduit. All the parameters taken for these models are detailed in the caption of the Figure 8 . In the far field, at distances greater than $0.2 L_{c}$ from the volcanic vent, magma pressure changes or dome height variations induce larger displacements at the Earth's surface than the magma conduit flow. Emplacement of a plug at the top of the conduit might induce larger amplitudes than magma reservoir or lava dome processes, in radial displacements as well as in tilt signal, only in the immediate vicinity of the vent.

To conclude, with the context of an incompressible magma, ground motion induced by magma viscosity increases at the top of the volcanic conduit will be detectable only if: (i) instruments are located in the near field, few hundred meters from the volcanic vent ; (ii) plugs have reduced size and high viscosity contrast compared to the magma column ; (iii) other processes, such as magma reservoir pressure changes or lava dome growth/collapse, do not dominate the deformation signal. Melnik and Sparks [2005] proposed a transient model of magma flow in an open volcanic conduit including gas exsolution and escape, bubble growth as well as crystallization effects. They show that the system can fluctuate between two stable states, one being characterised by a high flow rate of less viscous magma and the other by a low flow rate of more viscous magma. Considering the given viscosity profiles and reservoir overpressures, we have quantified the induced displacements when the system goes from one regime to the other. Deformation at the surface is almost 
entirely due to the pressure changes occurring within the reservoir, the viscosity profile changes occurring within the conduit having a smaller effect. However the model proposed by Melnik and Sparks [2005] cannot simultaneously explain cycles occurring on a period of several years or several weeks. Short time-scales cycles have been explained by models with a constant reservoir pressure [Costa et al., 2007b], which justifies our choice to quantify the displacement field induced by magma flow condition within the conduit in case there is no magma reservoir pressure change.

Conversely, the fact that conduit effects might dominate the deformation signal in the immediate vicinity of the vent, implies the necessity to have some distant instruments in order to monitor magma pressure changes within storage zones.

\subsection{Influence of Topography}

In all previous calculations, the volcano surface is modelled as a flat surface, but andesitic strato-volcanoes are characterized by significant topography with slopes up to $35^{\circ}$. Because topography can have an effect on ground deformation results [Cayol and Cornet, 1998], we calculated tilts induced by plug emplacement with an upper surface corresponding, in the first approximation (a linear trend), to Montserrat topography. Including this topography, we obtained the same conclusions as for a flat topography,

\subsection{Influence of Viscosity Profile}

At Soufrière Hills Volcano (Montserrat), tilt cycles were recorded at 600-700 m from the crater vent, first in December 1996 with an amplitude of 1-2 $\mu \mathrm{rad}$ and a period of 6-8 h, and few months after, from April to May 1997, with an amplitude between 10 and 25 urad and a period of 12-18 h [Voight et al., 1998]. The cyclic behaviour of the surface 
deformation, with a deflation phase more rapid than the inflation one, is also correlated with cycles in seismic activity and gas emissions of $\mathrm{SO}_{2}$ [Watson et al., 2000]. At Mt St Helens, variations of the tilt amplitude were also measured during the active period between the years 2004 and 2008 by Anderson et al. [2010]. Tiltmeters, located within the crater and close the dome (around 150-250 $\mathrm{m}$ from the vent), recorded many cyclic tilt events characterized by a duration from minutes to hours and a mean amplitude close to $1 \mu \mathrm{rad}$. The pattern of the cycle begin with, a rapid inflation (outward tilt) followed by a gradual subsidence (inward tilt). Here, we compare the amplitude of tilt recorded at Montserrat and Mt St Helens with those obtained by our plug models (Fig. 9b). Assuming a value of $0.8 \mathrm{GPa}$ for the shear modulus, 0.25 for the Poisson's ratio and $10 \mathrm{MPa}$ for the magma reservoir overpressure, a high viscous plug emplaced in the upper part of the conduit can produce the amplitude of tilt cycles recorded at both volcanoes. At Mt St Helens, surface tilt induced by a plug occupying $25 \%$ of the conduit fits the data collected during the period July 1996-January 1997. In the same way, at Soufrière Hills, a plug emplaced in the upper $10 \%$ of the conduit can also explain the tilt data recorded during December 1996. However, our plug models cannot produce the large tilt amplitude of 10-25 $\mu \mathrm{rad}$ measured during the April-May 1997 at Montserrat. In our model, we always assumed that viscosity increase at the top of the conduit follows a step function and we consider that the magma density is constant over the conduit. But, this viscosity profile is simplified and magma density also evolves with depth. To overcome this simplification, we also calculated tilt induced by other flow models considering realistic viscosity profiles, and compare to results previously obtained with our plug model. The first one is taken from Sparks [1997] and corresponds to the model 3 presented in the paper as a case "where 
a lava dome up to $100 \mathrm{~m}$ thick is being fed from a conduit and the gas is lost during ascent for magma that just remains gas-saturated at the local pressure". In this model, the viscosity profile is expressed as a power law of the depth and increases progressively from the bottom of the conduit to the surface. We solved the Navier-Stokes equations for an incompressible magma using this viscosity profile and applied the resulting stress at the conduit wall in order to calculate the displacement field. The other viscosity profile considered is obtained from Collombet [2009]. We used the stationary solution for flow conditions described by Collombet [2009] and applied the resulting stress at the conduit wall. In comparison with the previous models that only consider vertical gas escape, it also takes into account the lateral gas loss due to the permeability of the conduit wall. This model is suitable to model effusive activity, such as dome construction of andesitic strato-volcanoes because it allows to consider realistic initial volatiles content and to reproduce quite precisely the vesicularity of the degassed magma, which is consistent with the observations made on real domes. With this model, a large viscosity change occurs only in the upper few hundred metres of the conduit, which induces large stresses at the conduit wall close to the surface (Fig. 9a). The particularity of this last model is that, for shallow depths (above $750 \mathrm{~m}$ depth), magma overpressure in the conduit reaches larger values than those applied at the conduit bottom, due to magma compressibility effects. This large pressure gradient as well as the high shear stress at shallow levels of the conduit produced a tilt amplitude one order of magnitude larger than the two other models (Fig. 9b). Assuming a value of $0.8 \mathrm{GPa}$ for the shear modulus and $10 \mathrm{MPa}$ for the magma reservoir overpressure, the model obtained from Collombet [2009], taking into account a compressible magma and the vertical as well as the lateral degassing, seems 
than the plug length $\left(h_{p}\right)$ must be less than $7.5 \mathrm{~m}$ to reach the magma failure. Burgisser et al. [2011] suggest a thickness of a few tens of meters or even less for the dome plug, such that a thin plug might be possible. If we know consider a plug size of $50 \mathrm{~m}$, a bottom overpressure larger than the $66.7 \mathrm{MPa}$ is required for a $15 \mathrm{~m}$ radius conduit. In the case of a more realistic overpressure of $10 \mathrm{MPa}$, the brittle failure of the magma is obtained if the conduit radius is larger than $100 \mathrm{~m}$. Based on these results, conditions required to cause magma failure remain unlikely, such that we used a no-slip condition at the conduit wall. However, there is some experimental as well as textural evidence of magma brittle failure in the upper portion of volcanic conduits [Lavallée et al., 2008; Tuffen et al., 2008]. The value of shear strength we used might be overestimated for real magma and our Newtonian flow model does not well describe the stress gradient close to the conduit where the deformation is expected to be very localised.

Another way to account for plug removal is to consider a modification of permeability at the conduit wall as proposed by Edmonds et al. [2003]. For example, Taisne and Jaupart [2008] have shown that the loading of the crater floor by the dome acts to prevent gas leakage from magma by closing fractures around the volcanic conduit. A permeability decrease would prevent lateral degassing and might reduce the plug either by decreasing its viscosity contrast or its length. This is consistent with the fact that, as at Soufrière Hills (Montserrat), a decrease in $\mathrm{SO}_{2}$ emission rate was observed prior to the start of magma ascent, after a pause in dome growth [Edmonds et al., 2003]. In this case, the evolution of the plug, from one state of equilibrium to another, requires more time than in the case of slip by rupture. Based on the flow rate and plug geometry, we can calculate the amount of time required for the entire extrusion of the plug. This value is represented 
removed $\left(\frac{h_{p}}{L_{c}}=0\right)$. Because the ground movement has changed in direction, the surface of the volcano now tilts towards the vent. As a result, the process of thickness variation development of a viscous plug in a volcanic conduit can be a possible origin for the tilt reversal observed before an eruption at andesitic volcanoes, such as Mount St Helens.

\section{Conclusions}

We estimated ground deformation induced at a volcano surface by magma viscosity changes occurring in the upper portion of the conduit. Calculations were performed considering a simplified viscosity profile for the magma, the viscosity taking two different values: a higher value within the plug and a lower value within the magma column itself, below. This model provides a good estimation of ground displacement, not very different, in amplitude, from the one produced by more realistic models with power-law profiles of viscosity. Plug emplacement results in a decrease of the magma discharge rate together with a rise of the shear stress levels along the conduit walls, which is considered to be the main source of displacements at the surface. However, even if it is most often dominant, the shear stress effect is not the only effect of importance: the conduit is also pressurized all along the conduit, the maximum of pressurization occurring at the plug bottom. This pressurization has a significant effect on surface deformation and should not be neglected. For the case of Soufrière Hills Volcano at Montserrat, neglecting the pressure effects leads to an overestimation, larger than 20 percent, of the tilt measured at $500 \mathrm{~m}$ from the crater. Plug growth always results in an overall decreasing magma flow rate, whereas it may either induce an increase or a decrease of the outward tilt, depending on the magnitude and the depth of the pressurization level. Joint interpretation of magma 
flow rates and surface displacements can bring strong constrain on the plug thickness and viscosity evolution. In absence of dome load or magma reservoir pressure variations, radial displacements or tilt signals caused by plug emplacement might be detected only a few hundred metres from the crater vent, according to the size and the viscosity of the plug as well as the size of the conduit and the mechanical properties of the host rocks. This conclusion becomes even more restrictive in case the dome geometry evolves by collapse or rapid magma emplacement or in case magma reservoir pressure variations occur. Then a plug emplacement might dominates the tilt as well as radial displacements only in the immediate vicinity of the conduit (less than $100 \mathrm{~m}$ ). The estimated distance of detection should be taken into consideration when deciding for the type and the location of geodetic instrumentation at andesitic volcanoes. Or simplified model of plug emplacement can explain the amplitude around $1 \mu \mathrm{rad}$ of tilt signals recorded at Mt St Helens or Soufrière Hills Volcano at Montserrat in December 96. However, in order to explain the amplitude recorded, at Soufrière Hills, from May to August 2007, when the tilt reached more than 10 $\mu \mathrm{rad}$, we need to consider compressible magma characterized by a more realistic viscosity profile resulting from vertical as well as lateral degassing.

\section{Appendix A: Fluid-Solid Interaction}

In our study, we treat the full coupling between the fluid and the elastic solid by an iterative process. At each step, we solve for the fluid flow, apply the resulting stress components at the conduit wall, and then we calculate the displacements at the conduit walls and modify, in consequence, the geometry of the fluid domain boundaries. We iterate this process chain until convergence occurs. Usually convergence is immediate because the radial displacements of the conduit wall is small. This radial displacement can be 
approximated analytically. Neglecting the tangential stress effects and considering that the pressure gradient with depth remains small, we can use the solution of an infinite pressurised pipe with an axial geometry to estimate, to a first approximation, the radial

displacement of the conduit wall. It gives $U_{r}=\frac{1}{2}\left(\frac{P_{c}}{G}\right) a_{c}[$ Landau and Lifshitz, 1975; Love, 1987], where $P_{c}$ is the overpressure at the conduit bottom, $G$ the shear modulus of host rocks and $a_{c}$ the conduit radius. In volcanic conduits, the magma overpressure does not exceed values of $20 \mathrm{MPa}$ [Melnik and Sparks, 1999] and the shear modulus is around 1-10 GPa [Costa et al., 2007b; Voight et al., 1999; Barmin et al., 2002]. So, the amplitude, therefore, of the radial displacement $\left(U_{r}\right)$ at the conduit wall remains smaller than $1 \%$ of the conduit radius. For a 10 metre radius, the expected maximal wall displacement will be only 10 centimeters. We quantified, in our plug model, the effect of this wall deformation on the displacement field at the surface. First, wall deformation has a larger effect for a low shear modulus $(G)$ and a large overpressure $\left(P_{c}\right)$. Secondly, from a shear modulus of 0.4 GPa and a magma overpressure of $10 \mathrm{MPa}$, the amplitude of the surface displacements is only $5 \%$ larger when we take into account the conduit wall deformation compared with the rigid conduit case. In many volcanic contexts, the effect of conduit wall deformation will not affect significantly the surface displacements and can thus be neglected to a first approximation.

\section{Appendix B: Model validation}

Numerical solutions have been compared to existing analytic solutions in order to validate our model. First we used our numerical simulation to estimate the radial displacement induced, at the surface, by a pressurized pipe, with a uniform pressure $P_{c}$, embedded in an elastic half-space. Results were compared with the solution given by the following 
analytic expression [Love, 1987]: $U_{r}=\frac{a_{c}^{2} P_{c}}{2 r G}$, where $r$ is the radial distance. Secondly, the tilt induced, at the surface, by a constant shear stress applied along the conduit wall, was numerically estimated and compared to the analytic expression provided by Anderson et al. [2010], which corresponds to an approximate solution derived from the integration of the Green's functions of a vertical point force. The comparison between numerical results and analytic solutions as well as the estimated error are shown in Figure 11. The error is the relative difference in percent between the analytic calculation and our numerical results. This figure shows that the error is the largest at the smallest distance from the conduit. However, for a distance larger than 50 meters, the error remains smaller than 2 $\%$ for the pressurised pipe (case a) and close to 0 for the conduit with an applied shear stress (case b).

Acknowledgments. This study was supported by IRD. We are very grateful for helpful reviews from the Associate Editor M. Ryan, A. Costa, V. Cayol and an anonymous reviewer.

\section{References}

Anderson, K., M. Lisowski, and P. Segall, Cyclic ground tilt associated with the 2004-2008 eruption of mount St. Helens, J. Geophys. Res., 115, 2010.

Barmin, A., O. Melnik, and R. Sparks, Periodic behavior in lava dome eruptions, Earth Planet. Sci. Lett., 199, 173-184, 2002.

Beauducel, F., F.-H. Cornet, E. Suhanto, T. Duquesnoy, and M. Kasser, Constraints on magma flux from displacements data at Merapi volcano, Java, Indonesia, J. Geophys. Res., 105, 8193-8203, 2000. 
Bonaccorso, A., and P. Davis, Models of ground deformation from vertical conduits with application to eruptions of Mount St. Helens and Mount Etna, J. Geophys. Res., 104, 10,531-10,542, 1999.

Burgisser, A., S. Poussineau, L. Arbaret, T. Druitt, T. Giachetti, and J.-L. Bourdier, Pre-explosive conduit conditions of the 1997 Vulcanian explosions at Soufrière Hills Volcano, Montserrat: I. Pressure and vesicularity distributions, J. Volcanol. Geotherm. Res., 194, 27-41, 2010.

Burgisser, A., L. Arbaret, T. Druitt, and T. Giachetti, Pre-explosive conduit conditions of the 1997 Vulcanian explosions at Soufrière Hills Volcano, Montserrat: II. Overpressure and depth distributions, J. Volcanol. Geotherm. Res., 199, 193-205, 2011.

Caricchi, L., L. Burlini, P. Ulmer, T. Gerya, M. Vassalli, and P. Papale, Non-Newtonian rheology of crystal-bearing magmas and implications for magma ascent dynamics, Earth Planet. Sci. Lett., 264, 402-419, 2007.

Cayol, V., and F. Cornet, Effects of topography on the interpretation of the deformation field of prominent volcanoes - Application to Etna, Geophys. Res. Letters, 25, 1979$1982,1998$.

Chadwick, W., R. Archuleta, and D. Swanson, The Mechanics of Ground Deformation Precursory to Dome-Building Extrusions at Mount St. Helens 1981-1982, J. Geophys. Res., 93, 4351-4366, 1988.

Collier, L., and J. Neuberg, Incorporating seismic observations into 2D conduit flow modeling, J. Volcanol. Geotherm. Res., 152, 331-346, 2006.

Collombet, M., Two-dimensional gas loss for silicic magma flows: toward more realistic numerical models, Geophys. J. Int., 177, 309-318, 2009. 
Costa, A., and G. Macedonio, Viscous heating in fluids with temperature-dependent viscosity: Triggering of secondary flows, J. Fluid Mech., 540, 21-38, 2005.

Costa, A., O. Melnik, and R. Sparks, Controls of conduit geometry and wallrock elasticity on lava dome eruptions, EPSL, 260, 137-151, 2007a.

Costa, A., O. Melnik, R. Sparks, and B. Voight, Control of magma flow in dykes on cyclic lava dome extrusion, Geophys. Res. Letters, 34, L02,303, 2007b.

Costa, A., O. Melnik, and E. Vedeneeva, Thermal effects during magma ascent in conduits, J. Geophys. Res., 112, 2007c.

Denlinger, R., and R. Hoblitt, Cyclic eruptive behavior of silicic volcanoes, Geology, 27, 459-462, 1999.

Edmonds, M., C. Oppenheimer, D. Pyle, R. Herd, and G. Thompson, $\mathrm{SO}_{2}$ emissions from Soufrière Hills Volcano and their relationship to conduit permeability, hydrothermal interaction and degassing regime, J. Volcanol. Geotherm. Res., 124, 23-43, 2003.

Gardner, J., R. Thomas, C. Jaupart, and S. Tait, Fragmentation of magma during Plinian volcanic eruptions, Bull. Volcanol., 58, 144-162, 1996.

Green, D., J. Neuberg, and V. Cayol, Shear stress along the conduit wall as a plausible source of tilt at Soufrière Hills volcano, Montserrat, Geophys. Res. Letters, 33, L10,306, 2006.

Harris, A., W. Rose, and L. Flynn, Temporal trends in lava dome extrusion at Santiaguito 1922-2000, 65, 77-89, 2002.

Hautmann, S., J. Gottsmann, R. Sparks, A. Costa, O. Melnik, and B. Voight, Modelling ground deformation caused by oscillating overpressure in a dyke conduit at Soufrière Hills Volcano, Montserrat, Tectonophysics, 471, 87-95, 2009. 
Hess, K.-U., and D. Dingwell, Viscosities of hydrous leucogranitic melts: A non-Arrhenian model, American Mineralogist, 81, 1297-1300, 1996.

Iverson, R., et al., Dynamics of seismogenic volcanic extrusion at Mount St Helens in 2004-05, Nature, 444, 439-443, 2006.

Landau, L., and E. Lifshitz, Theory of elasticity, 2nd ed. ed., Pergamon, Tarrytown, N.Y., 1975.

Lavallée, Y., P. Meredith, D. Dingwell, K.-U. Hess, J. Wassermann, B. Cordonnier, A. Gerik, and J. Kruhl, Seismogenic lavas and explosive eruption forecasting, Nature, 453, 507-509, 2008.

Lejeune, A., and P. Richet, Rheology of crystal-bearing silicate melts: An experimental study at high viscosities, J. Geophys. Res., 100, 4215-4229, 1995.

Lensky, N., R. Sparks, O. Navon, and V. Lyakhovsky, Cyclic activity at Soufrière Hills Volcano, Montserrat: degassing-induced pressurization and stick-slip extrusion, in Fluid Motions in Volcanic Conduits: A Source of Seismic and Acoustic Signals, vol. 307, Geological Society ed., pp. 169-188, Lane, S.J. \& Gilbert, J.S., London, 2008.

Lister, J., and R. Kerr, Fluid-Mechanical models of crack propagation and their application to magma transport in dikes, J. Geophys. Res., 96, 10,049-10,077, 1991, archimede.

Llewellin, E., and M. Manga, Bubble suspension rheology and implications for conduit flow, J. Volcanol. Geotherm. Res., 143, 205-217, 2005.

Love, A., A Treatise on the mathematical theory of elasticity, Dover Classics of Sciences and Mathematics, 4th ed., Dover, 1987.

Mastin, G., and D. Pollard, Surface Deformation and Shallow Dike Intrusion Processes at Inyo Craters, Long Valley, California, J. Geophys. Res., 93, 13,221-13,235, 1988. 
Melnik, O., and R. Sparks, Nonlinear dynamics of lava dome extrusion, Nature, 402, 37-41, 1999.

Melnik, O., and R. Sparks, Controls on conduit magma flow dynamics during lava dome building eruptions, J. Geophys. Res., 110, 2005.

Mogi, K., Relations between the eruptions of various volcanoes and the deformation of the ground surfaces around them, Bull. Earthquake Res. Inst., Univ. Tokyo, 36, 99-134, 1958, analytic.

Nakada, S., and J. Eichelberger, Looking into a volcano: drilling Unzen, Geotimes, 49, $14-17,2004$.

Nakada, S., H. Shimizu, and K. Ohta, Overview of the 1990-1995 eruption at Unzen Volcano, J. Volcanol. Geotherm. Res., 89, 1-22, 1999.

Nishimura, T., Ground deformation due to magma ascent with and without degassing, Geophys. Res. Letters, 33, L23,309, 2006.

Nishimura, T., Ground deformation caused by magma ascent in an open conduit, $J$. Volcanol. Geotherm. Res., 187, 178-192, 2009.

Pinkerton, H., and R. Stevenson, Methods of determining the rheological properties of magmas at sub-liquidus temperatures, J. Volcanol. Geotherm. Res., 53, 47-66, 1992.

Roman, D., J. Neuberg, and R. Luckett, Assessing the likelihood of volcanic eruption through analysis of volcanotectonic earthquake fault-plane solutions, Earth Planet. Sci. Lett., 248, 244-252, 2006.

Rubin, A., Propagation of magma-filled cracks, Ann. Rev. Earth Planet. Sci., 23, 287-336, 1995. 
Shaw, H., Obsidian-H20 Viscosities at 1000 and 2000 Bars in the Temperature Range $700^{\circ}$ to $900^{\circ} \mathrm{C}$, J. Geophys. Res., 68, 6337-6343, 1963.

Sparks, R., Causes and consequences of pressurisation in lava dome eruptions, Earth Planet. Sci. Lett., 150, 177-189, 1997.

Sparks, R., and S. Young, The eruption of Soufrière Hills volcano, Montserrat (1995-1999): overview of scientific results, in The eruption of the Soufrière Hills Volcano, Montserrat from 1995 to 1999, edited by T. Druitt and B. Kokelaar, pp. 45-69, Geological Society, 2002.

Sparks, R., M. Murphy, A. Lejeune, R. Watts, J. Barclay, and S. Young, Control on the emplacement of the andesite lava dome of the Soufrière Hills Volcano, Montserrat by degassing-induced crystallization, Terra Nova, 12, 14-20, 2000.

Swanson, D., and R. Holcomb, Regularities in growth of the Mount St. Helens dacite dome 1980-1986, in Lava Flows and Domes: Emplacement and Mechanisms and Hazards Implications, Springer ed., pp. 3-24, J.H. Fink, New York, 1990.

Taisne, B., and C. Jaupart, Magma Degassing and Intermittent Lava Dome Growth, Geophys. Res. Letters, 35, 2008.

Tuffen, H., and D. Dingwell, Fault textures in volcanic conduits: evidence for seimic trigger mechanisms during silicic eruptions, Bull. Volcanol., 67, 370-387, 2005.

Tuffen, H., D. Dingwell, and H. Pinkerton, Repeated fracture and healing of silicic magma generate flow banding and earthquakes?, Geology, 31, 1089-1092, 2003.

Tuffen, H., R. Smith, and R. Sammonds, Evidence for seismogenic fracture of silicic magma, Nature, 453, 511-513, 2008. 
Voight, B., R. Hoblitt, A. Clarke, A. Lockhart, A. Miller, L. Lynch, and J. McMahon, Remarkable cyclic ground deformation monitored in real-time on Montserrat, and its use in eruption forecasting, Geophys. Res. Letters, 25, 3405-3408, 1998.

Voight, B., E. Constantine, S. Siswowidjoyo, and R. Torley, Historical eruptions of Merapi Volcano, central Java, Indonesia, 1768-1998, J. Volcanol. Geotherm. Res., 100, 69-138, 2000 .

Voight, B., et al., Magma Flow Instability and Cyclic Activity at Soufriere Hills Volcano, Montserrat, British West Indies, Science, 283, 1999.

Voight, B., et al., Unprecedented pressure increase in deep magma reservoir triggered by lava-dome collapse, Geophys. Res. Letters, 33, 2006.

Watson, I., et al., The relationship between degassing and ground deformation at Soufriere Hills Volcano, Montserrat, J. Volcanol. Geotherm. Res., 98, 117-126, 2000.

Webb, S., and D. Dingwell, Non-Newtonian Rheology of Igneous Melts at High Stresses and Strain Rates: Experimental Results for Rhyolite, Andesite, Basalt, and Nephelinite, J. Geophys. Res., 95, 15,695-15,701, 1990.

Williams, P., and S. Self, The October 1902 Plinian eruption of Santa Maria volcano, Guatemala, J. Volcanol. Geotherm. Res., 16, 1983.

Wylie, J., B. Voight, and J. Whitehead, Instability of Magma Flow from VolatileDependent Viscosity, Science, 285, 1999.

Yokoyama, I., Geophysical characteristics of dacite volcanism 1977-1978 eruption of Usu volcano, J. Volcanol. Geotherm. Res., 9, 335-358, 1981. 
Figure 1. a) Schematic representation of the plugged conduit model. Geometric and physical parameters are shown. No displacement in the direction perpendicular to the surface is allowed at the right and lower boundaries. The upper boundary is a free surface. The right boundary is situated far away from the conduit (at $100 \mathrm{~km}$ ) in order to prevent boundary effects. Fluid flow is caused by pressure difference between the conduit bottom $\left(P=P_{c}\right)$ and the surface $(P=0)$. A no-slip condition is applied at the fluid along the conduit wall. The continuity of the stress field, normal as well as tangential components, is applied at the conduit wall. The formation of the plug at the top of the conduit is modelled by an increase of the magma viscosity. b) 1-D viscosity profile used in our calculation (left side) and the resulting stresses (pressure and shear stress) at the conduit wall (right side). As a first approximation, the increase of the viscosity is modelled by a "step" function, with two extreme values: the viscosity in the magma column below the plug, $\mu_{m}$, and the viscosity of the plug, $\mu_{p}$. We consider an incompressible magma with constant density. In each sketch, dashed curves represented our reference case: the Poiseuille flow with a constant viscosity $\left(\mu=\mu_{m}\right.$ at any depth). 


\section{Figure 2.}

a) Mesh of the numerical model superimposed to the pattern of total displacements induced within the host rocks by a Poiseuille magma flow. The mesh is highly refined in the region close to the conduit, where the gradient of displacements are the largest. Displacements are induced by a flow at constant viscosity initiated by a pressure of $10 \mathrm{MPa}$ at the bottom of a conduit. The conduit is a cylinder with a $15 \mathrm{~m}$ radius and a $5000 \mathrm{~m}$ length. Elastic parameters of the host rocks are respectively $4 \mathrm{GPa}$ and 0.25 for the shear modulus $G$ and the Poisson's ratio $\nu$. Note that in lateral extension only the first $10 \mathrm{~km}$ of the $100 \mathrm{~km}$ corresponding to the actual box size, are shown. b) Zoom of the mesh in the $1 \mathrm{~km}^{2}$ area near the surface corresponding to the red box of Figure a).

Figure 3. Surface displacements: radial (a), vertical (b) and tilt (c) induced by a constant viscosity magma flow (reference case chosen in the following). Displacements are obtained with respect to the lithostatic medium containing an overpressurized reservoir not connected to the surface with a Poisson's ratio equal to 0.25. Results are presented for three different conduit

aspect ratio $\left(\frac{a_{c}}{L_{c}}\right)$. Distances are normalized by the conduit length $\left(L_{c}\right)$ and displacement by the ratio $\left(\frac{P_{c}}{G} L_{c}\right)$. The distance $\mathrm{r}=0$ corresponds to the conduit wall. In each case, the total displacement (solid line) as well as displacement only induced by the shear stress component (short dashed) or by the pressure (large dashed) are shown. 
Figure 4. Tilt induced by the formation of a plug at the top of the conduit, shown as a function of the two dimensionless numbers characterizing the plug: the length ratio $\left(\frac{h_{p}}{L_{c}}\right)$ and the viscosity ratio $\left(\frac{\mu_{p}}{\mu_{m}}\right)$. Tilt is calculated, at the surface, $500 \mathrm{~m}$ away from the conduit wall. Results are shown for a given conduit geometry, with a radius $\left(a_{c}\right)$ of $15 \mathrm{~m}$ and a length $\left(L_{c}\right)$ of $5000 \mathrm{~m}$. The pressure $P_{c}$ applied at the bottom is fixed at $10 \mathrm{MPa}$, the shear modulus of the host rocks, $G$, is equal to $0.8 \mathrm{GPa}$ and the Poisson's ratio is set to 0.25 . a) Part of the tilt which is only induced by the shear stress applied at the conduit wall. b) Part of the tilt which is only induced by the pressure applied at the conduit wall. Tilt represented in c) is the summation of the values shown in b) and c). The dashed line corresponds to the critical plug thickness, $h_{p(\text { crit })}$, which gives the maximal tilt value for a given viscosity ratio. Note that tilt is relative to our reference case obtained with a constant viscosity magma flow, which means that tilt tends to zero when either $h_{p}$ tends to zero or the viscosity ratio $\frac{\mu_{p}}{\mu_{m}}$ tends to 1.

Figure 5. Detection distance for the horizontal (a) and vertical (b) displacements as a function of the two dimensionless numbers related to the plug: the length ratio $\left(\frac{h_{p}}{L_{c}}\right)$ and the viscosity ratio $\left(\frac{\mu_{p}}{\mu_{m}}\right)$. The detection distance here corresponds to the maximal radial distance from the conduit wall where, respectively, a horizontal and a vertical displacement larger in amplitude than, respectively, one $\mathrm{mm}$ and $1 \mathrm{~cm}$, is expected. The conduit length $\left(L_{c}\right)$ and the pressure $\left(P_{c}\right)$ applied at the bottom are, respectively, set to $5000 \mathrm{~m}$ and $10 \mathrm{MPa}$. The Poisson's ratio is set to 0.25 and $a_{c}=15 \mathrm{~m}$ and $G=0.8 \mathrm{GPa}$. 
Figure 6. Detection distance for the tilt signal, function of the two dimensionless numbers related to the plug: the length ratio $\left(\frac{h_{p}}{L_{c}}\right)$ and the viscosity ratio $\left(\frac{\mu_{p}}{\mu_{m}}\right)$. The detection distance here corresponds to the maximal radial distance from the conduit wall where a tilt larger in amplitude than one microradian is expected. The conduit length $\left(L_{c}\right)$ and the pressure $\left(P_{c}\right)$ applied at the bottom are, respectively, set to $5000 \mathrm{~m}$ and $10 \mathrm{MPa}$. The Poisson's ratio is set to 0.25. Results are presented for four different cases: a) $a_{c}=50 \mathrm{~m}$ and $G=0.8 \mathrm{GPa}$ b) $a_{c}=50$ $\mathrm{m}$ and $G=4.0 \mathrm{GPa}, \mathrm{c}) a_{c}=15 \mathrm{~m}$ and $G=0.8 \mathrm{GPa}$ and d) $a_{c}=15 \mathrm{~m}$ and $G=4.0 \mathrm{GPa}$.

Figure 7. Relationship between tilt signal, magma flow rate and extrusion duration during the evolution of a plug within the volcanic conduit. Conditions are, as for Figure 4, obtained for a conduit radius $\left(a_{c}\right)$ of $15 \mathrm{~m}$, a conduit length $\left(L_{c}\right)$ of $5000 \mathrm{~m}$, a bottom pressure $\left(P_{c}\right)$ of $10 \mathrm{MPa}$, a shear modulus $(G)$ equal to $0.8 \mathrm{GPa}$ and a Poisson's ratio $(\nu)$ set to 0.25 . Two end-members for the plug evolution are tested: (1) a viscosity increase of plugs having a given thickness (left panels) ; (2) a thickness increase for plugs having a constant viscosity (right panels). For each case, we show a) Tilt calculated at a radial distance of $500 \mathrm{~m}$ from the conduit ; b) Normalized magma flow rate $\left(Q / Q_{r e f}\right)$ deduce from the expression $\left.(3) ; c\right)$ Extrusion duration in hours, which corresponds to the amount of time required to totally extrude the plug considered (with thickness $h_{p}$ and viscosity $\mu_{p}$ ). This parameter is directly inferred from the ratio between the volume of the plug $\left(\pi a_{c}^{2} h_{p}\right)$ and the magma flux $Q$, when the value of the magma viscosity $\mu_{m}$ is set. Here, the extrusion duration is obtained taking $\mu_{m}=10^{4}$ Pa.s. 
Figure 8. Amplitude of surface ground motion : a) the radial and b) vertical displacements as well as c) the tilt for various processes occurring on andesitic strato-volcanoes, detailed on sketch d). Numerical calculations are performed with the COMSOL software, taking a value of 0.8 GPa for rocks rigidity and 0.25 for the Poisson's ratio. The reference state is an overpressurized reservoir $\left(P_{c}=\rho_{m} g L_{c}+10 \mathrm{MPa}\right)$ embedded in a lithostatic medium, which feeds an open conduit with length $\left(L_{c}\right)$ and radius $\left(a_{c}\right)$ respectively equal to $5000 \mathrm{~m}$ and $15 \mathrm{~m}$. Magma in the conduit has a constant viscosity $\left(\mu_{m}\right)$. Perturbations are the following: (1) Overpressure change of $\Delta P_{c}=1$ MPa due to magma replenishment or withdrawal within a $10 \mathrm{~km}^{3}$ spherical magma reservoir (long dashed curves in a-b-c). (2) Plug emplacement in the upper part of the conduit, characterised by

a thickness $\left(h_{p}\right)$ of $50 \mathrm{~m}$ and a viscosity ratio $\left(\frac{\mu_{p}}{\mu_{m}}\right)$ equal to $10^{5}$ (solid curves in a-b-c). (3) Load change of $P_{d}=1 \mathrm{MPa}$ due to the construction/destruction of a $200 \mathrm{~m}$ radius $\left(R_{d}\right)$ lava dome at the surface (short dashed curves in a-b-c). Note that the value of $1 \mathrm{MPa}$ can be associated to a dome height variation of $60 \mathrm{~m}$ for eruptive products density of $1700 \mathrm{~kg} \cdot \mathrm{m}^{-3}$. 
Figure 9. a) Magma viscosity as a function of normalized depth (left side) and resulting stress components along the conduit wall (right side) for various models. Note that stress components represented are the differential terms compared to the lithostatic stress field. Black lines correspond to plug models described in our study. Four models are tested with the same viscosity contrast $\left(\frac{\mu_{p}}{\mu_{m}}=10^{5}\right)$ but different length ratio: $\frac{h_{p}}{L_{c}}=0.01, \frac{h_{p}}{L_{c}}=0.05, \frac{h_{p}}{L_{c}}=0.10$ and $\frac{h_{p}}{L_{c}}=0.25$. Red and blue lines correspond to calculation with more realistic profile for magma viscosity, which depends on the gas escape process occurring on the magma column. The first (red) is derived from Sparks [1997], where depth dependence of magma viscosity is expressed as a power-law function. The second (blue), deduced from the study of Collombet [2009], considers vertical gas loss as the previous, but also the lateral gas escape due to wall permeability. b) Tilt signal at the surface induced by the different flow models discussed in a). The reference state is the Poiseuille flow with a constant viscosity. The blue boxes indicate the tilt amplitude recorded in two andesitic volcanoes: (1) and (2) at Soufrière Hills (Montserrat) respectively in May-August 1997 and December 1996 [Voight et al., 1998] ; (3) at Mt St Helens between July 2006 and January 2007 [Anderson et al., 2010]. Parameters used for the calculation are the same as in Figure 4. 
Figure 10. a) Evolution of the tilt calculated at $500 \mathrm{~m}$ from the conduit (black line) and the magma flow rate (grey line) during the plug thickness decrease. The viscosity ratio $\frac{\mu_{p}}{\mu_{m}}$ is fixed at 100. All parameters are the same as in Figure 7. The dots associated with numbers correspond to different cases of plug thickness, from 1 (very thick) to 4 (very thin). The last case, number 5, is related to a model without plug. b) Sketch showing the evolution of ground movement and magma flow rate during the decrease of a plug thickness. From case 3 to 4 , we see a change in the direction of ground movement (inflation to subsidence) correlated with a strong increase of the magma flux. Both changes could be used as precursors in the eruption forecasting of andesitic volcanoes.

Figure 11. Analytic (solid lines) against numerical solutions (dots) for ground surface motion: a) the radial displacements induced by a pressurized conduit and b) the tilt induced by a vertical traction along the conduit wall. The conduit is a cylinder with a $15 \mathrm{~m}$ radius and a $5000 \mathrm{~m}$ length. Elastic medium is characterized by a shear modulus of $0.8 \mathrm{GPa}$ and a Poisson's ratio of 0.25 . In a), all the conduit is pressurized with a uniform pressure equal to $10 \mathrm{MPa}$. In b), the shear stress equal to $3000 \mathrm{~Pa}$ is applied in the lower part of the conduit, between -2500 and $-5000 \mathrm{~m}$ depth. The bottom panels show for each case the relative difference in percent between analytic and numerical solutions. 
a) $\begin{gathered}\text { axis of } \\ \text { symm netry }\end{gathered}$

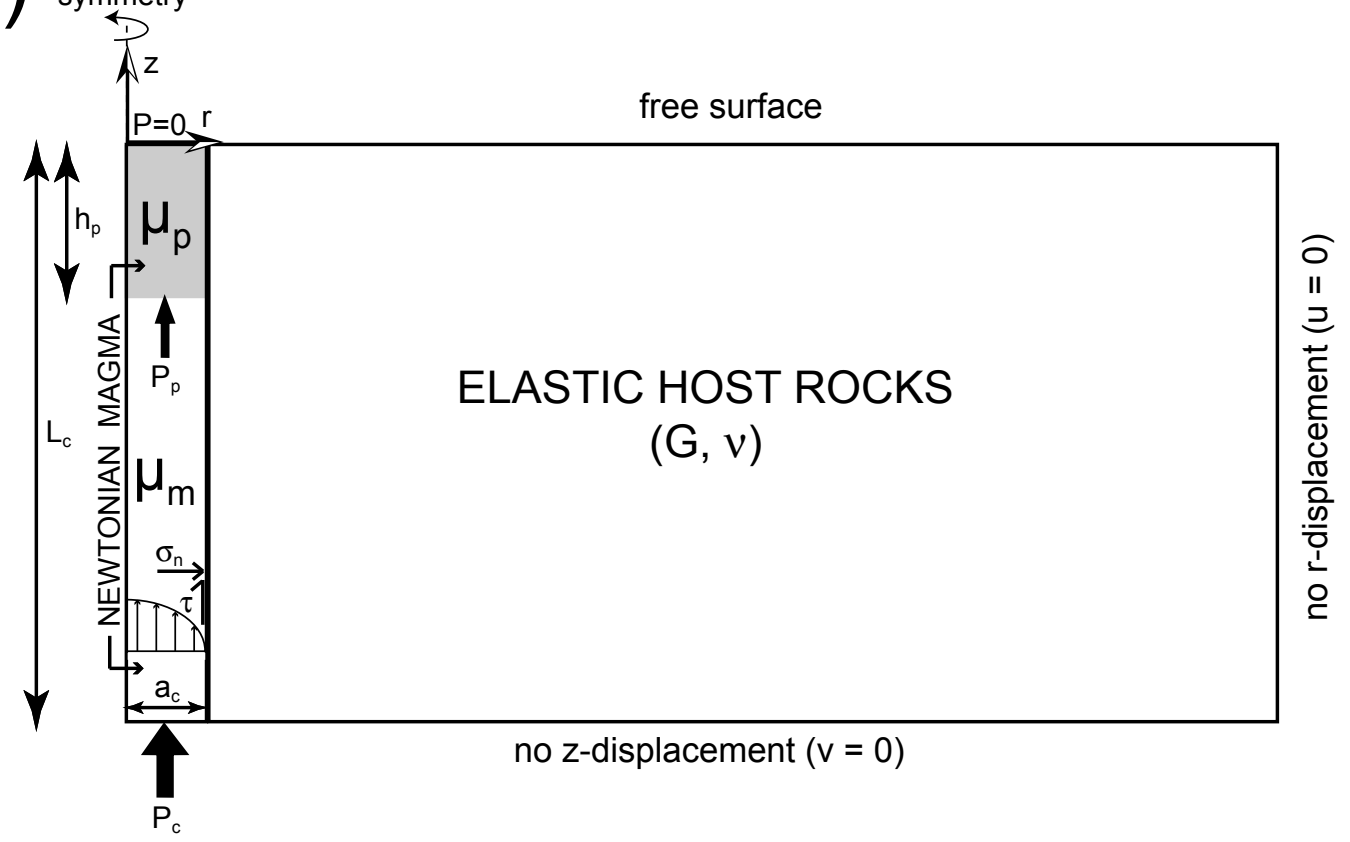

MAGMA VISCOSITY
PROFILE

STRESSES AT THE CONDUIT WALL

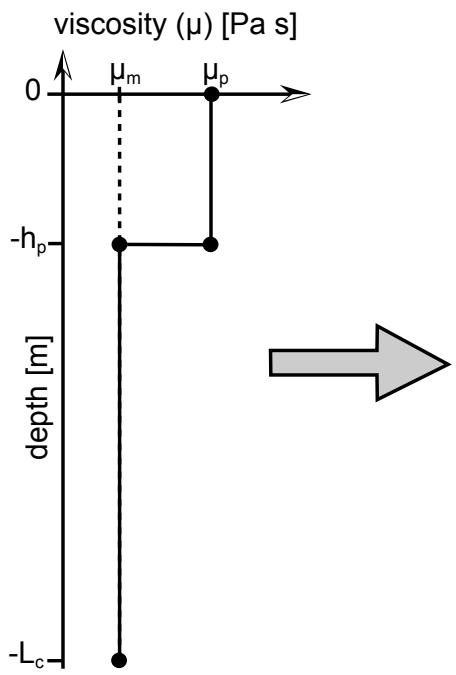

normal stress $\left(\sigma_{n}\right)=$ pressure $[\mathrm{Pa}]$ shear stress $(\tau)[\mathrm{Pa}]$
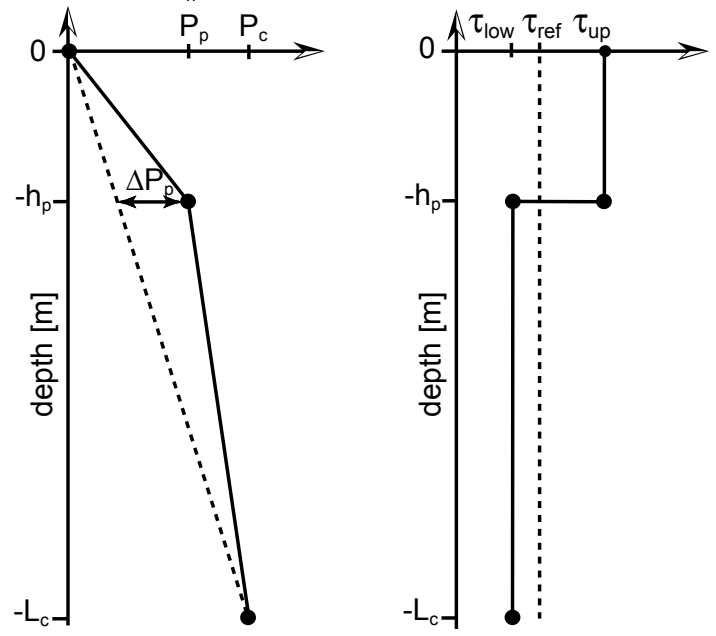


$$
\mathrm{a}_{\mathrm{c}} / \mathrm{L}_{\mathrm{c}}=0.1
$$

a)
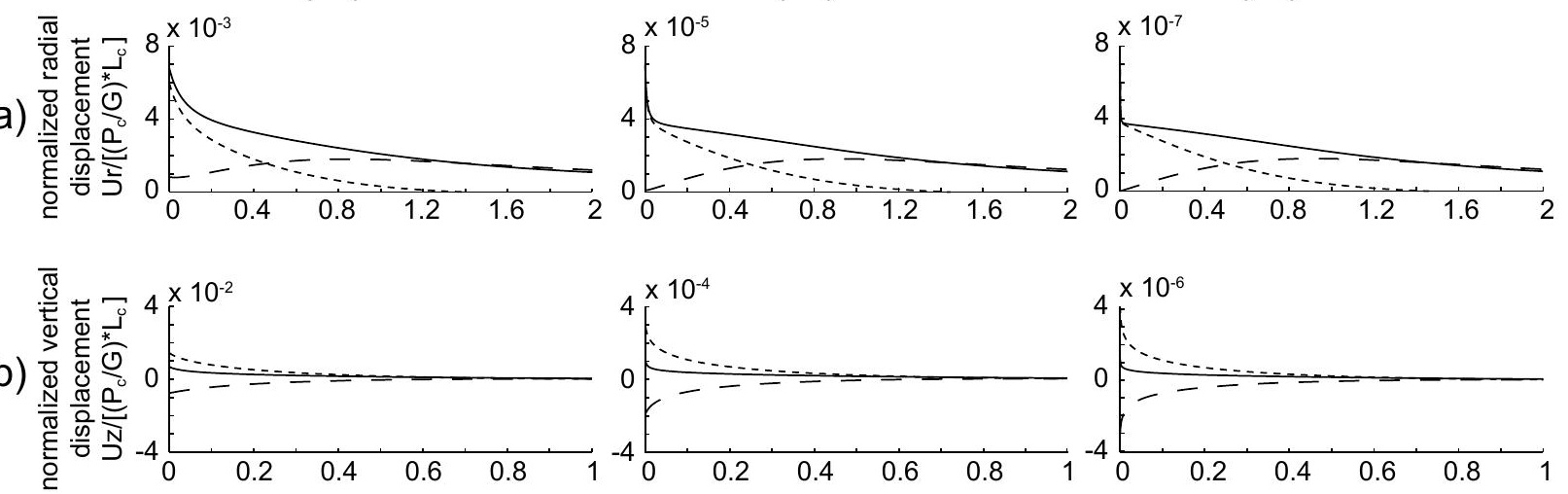

c)
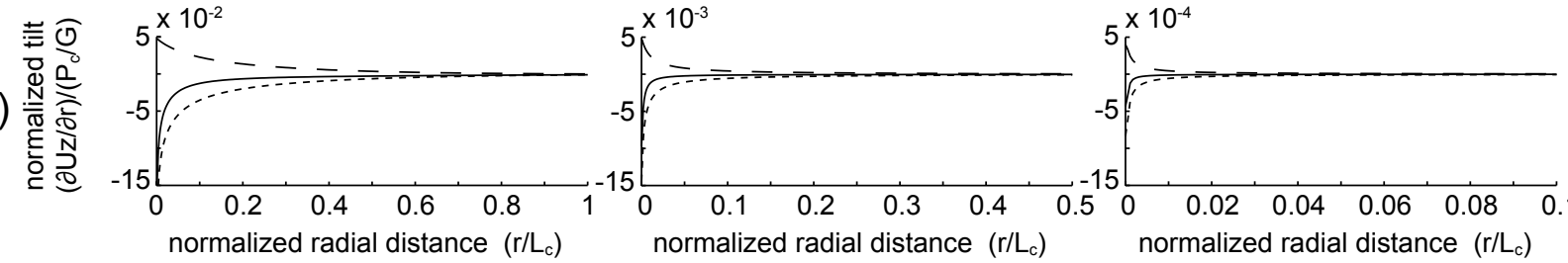

shear stress

- - - pressure shear stress + pressure 
a) Shear stress
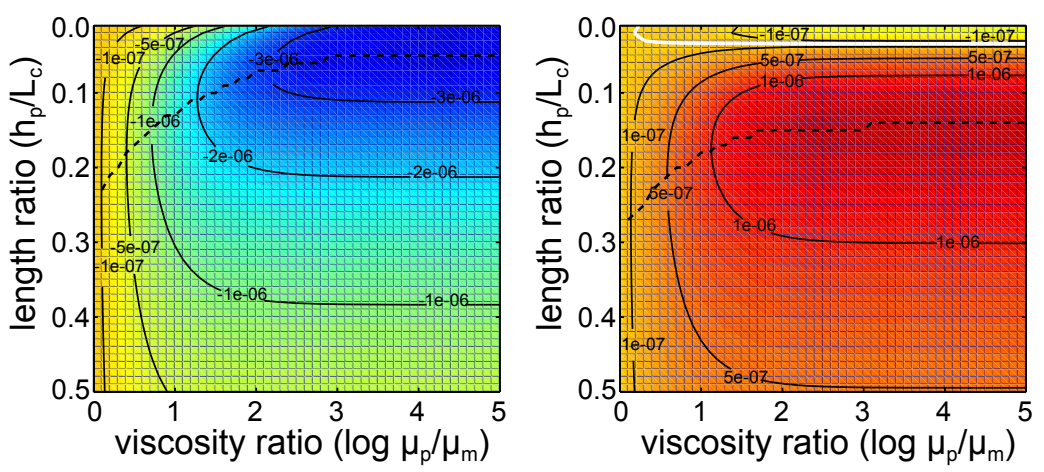

c) Shear + pressure

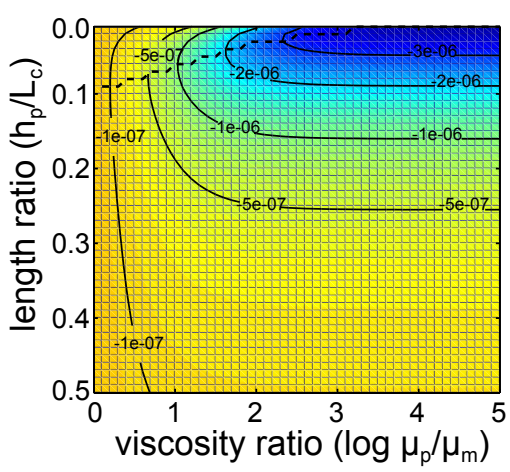

$\begin{array}{rrrrrrr}-4 & -3 & -1 & -2 & 0 & 1 & 2 \\ & & & & & & \\ {[\mathrm{rad}]} & \end{array}$


Horizontal displacement $\left(\mathrm{U}_{\mathrm{r}}\right)$

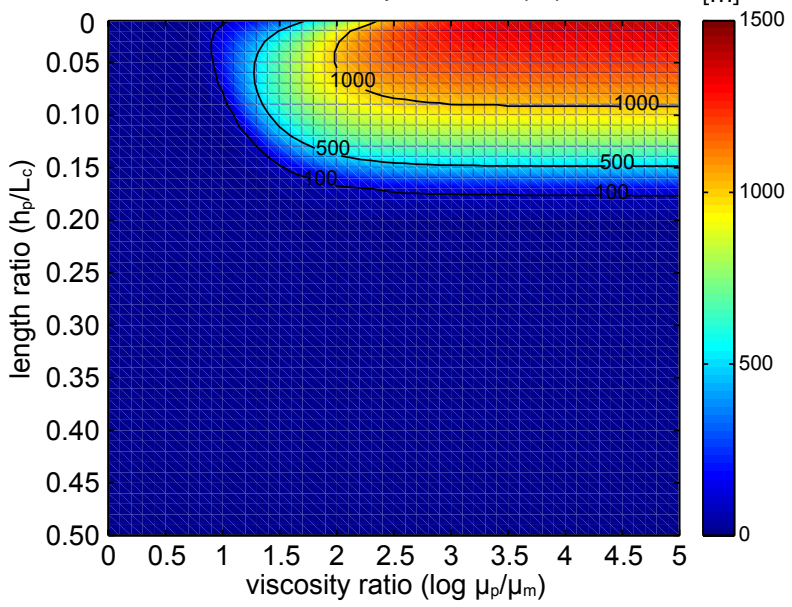

Vertical displacement $\left(\mathrm{U}_{\mathrm{z}}\right)$

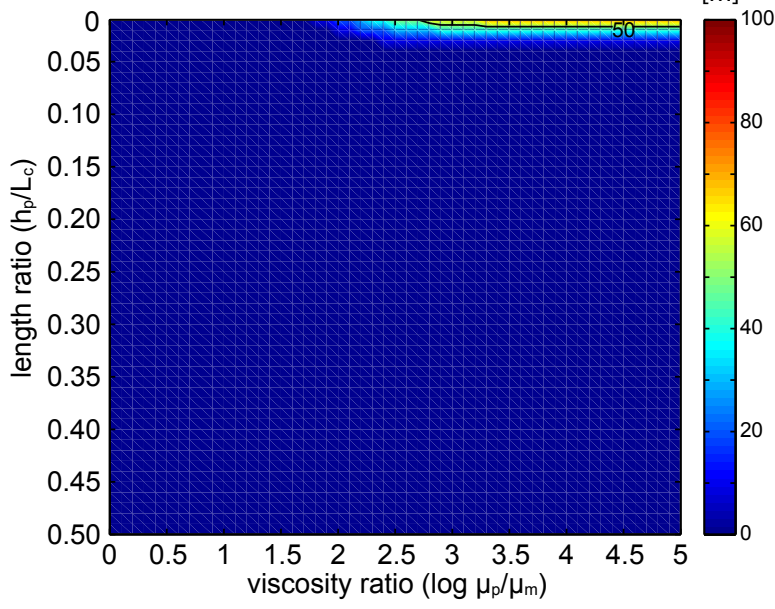


(1) Plug viscosity

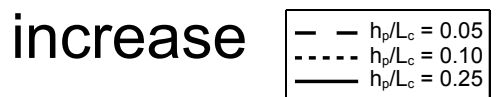
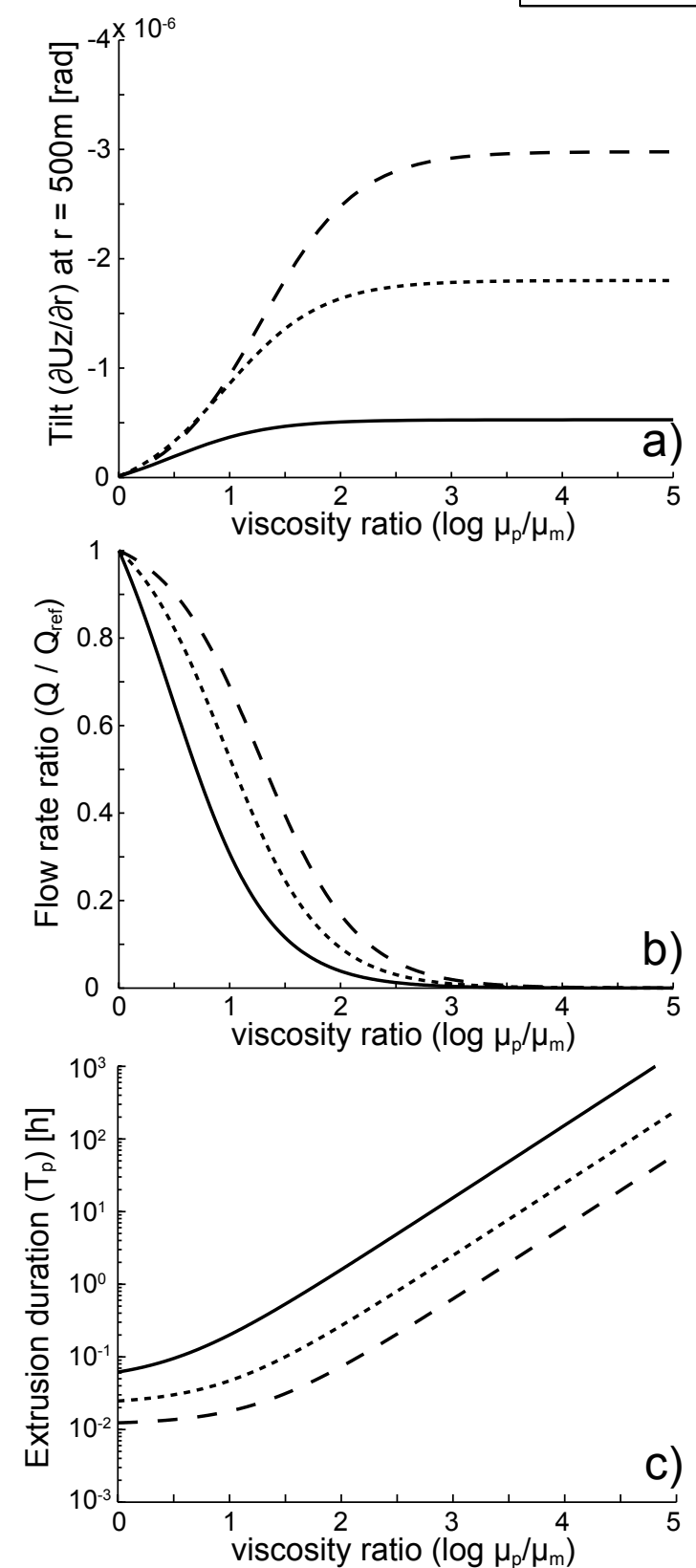

(2) Plug thickness increase
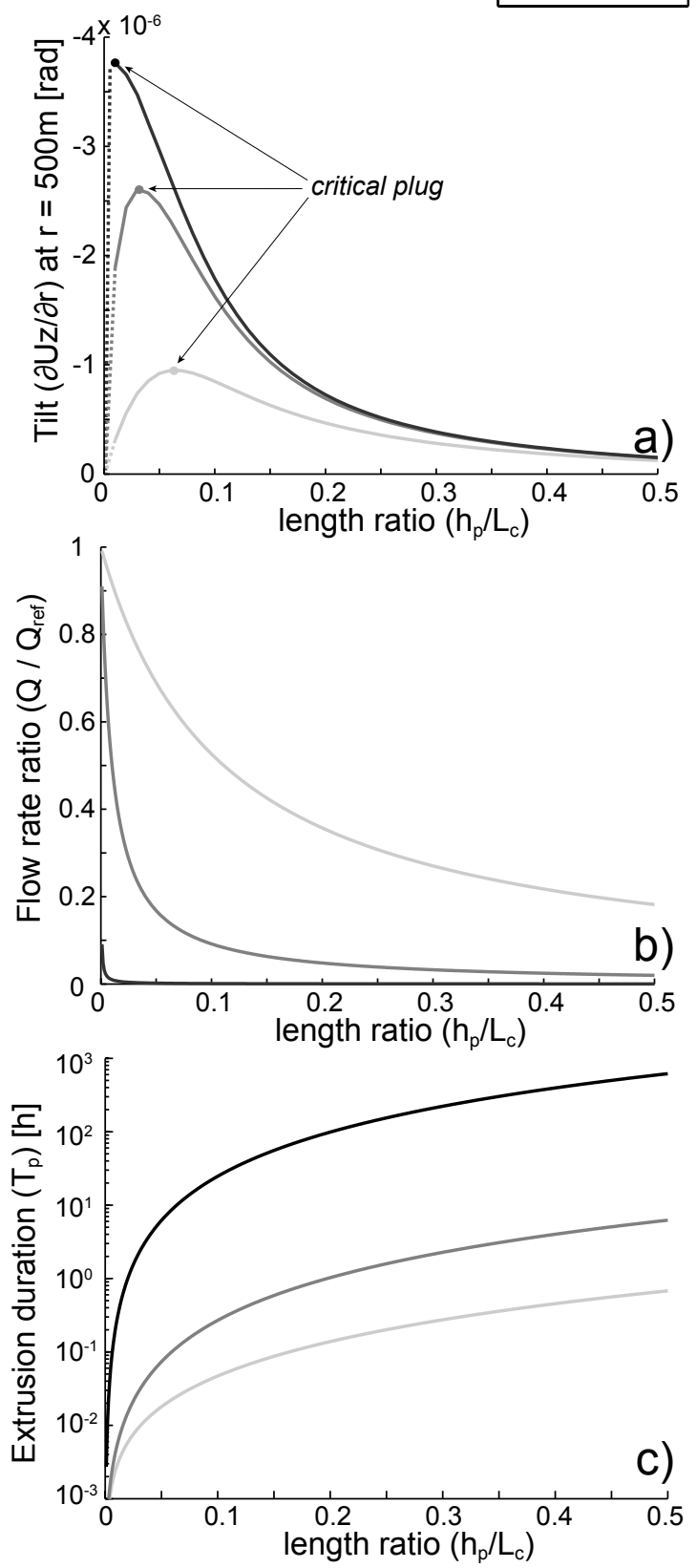


\section{a) Depth profile of the viscosity and the resulting stresses at the conduit wall}
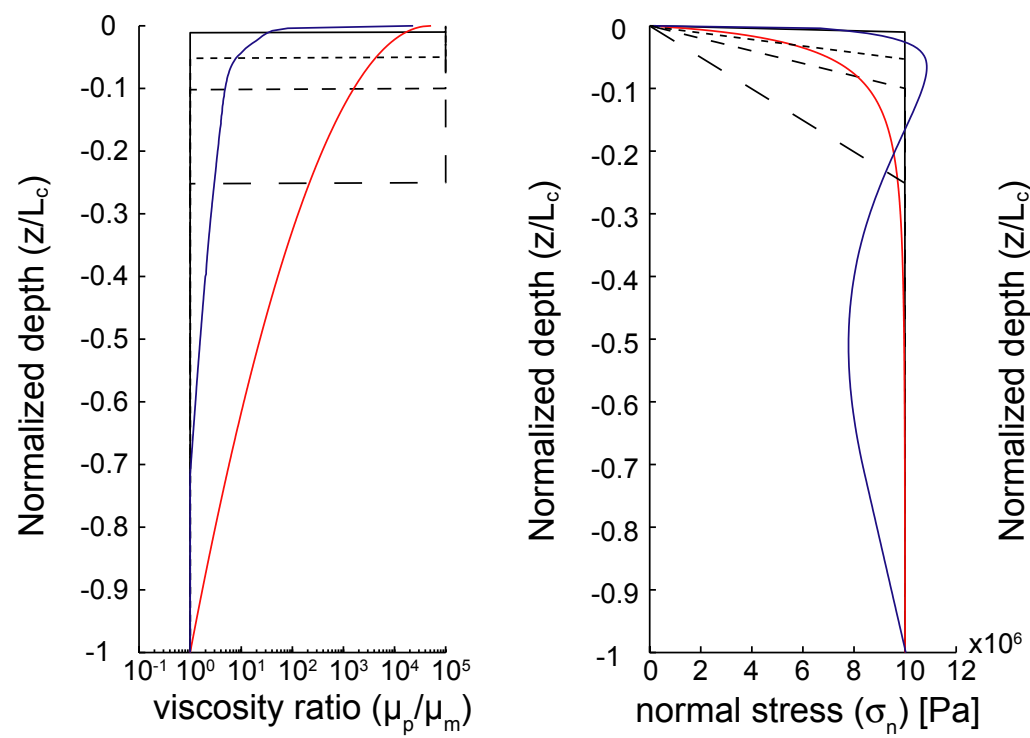

\section{b) Tilt at the surface}

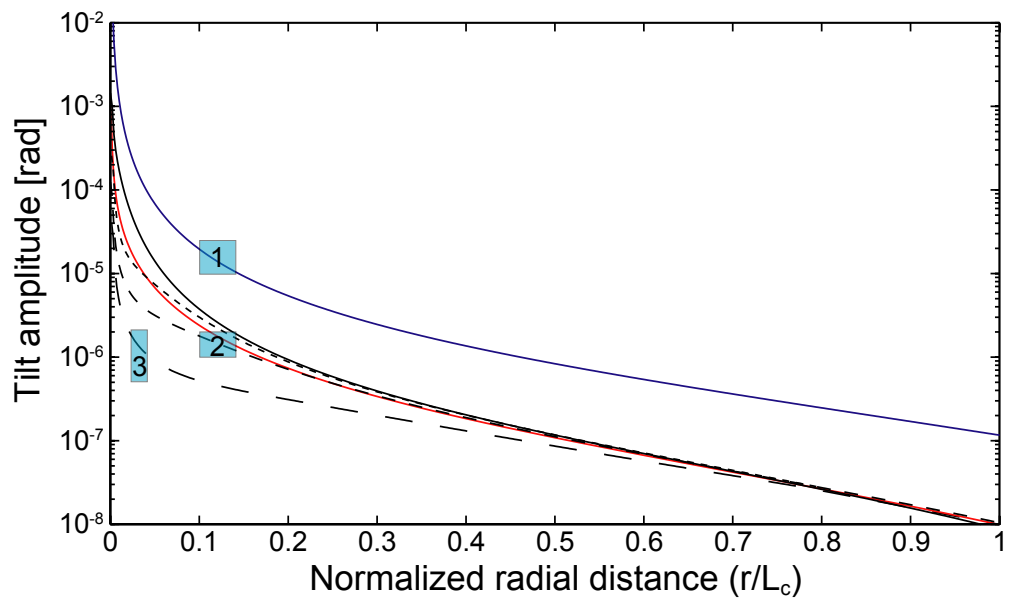

Our plug model

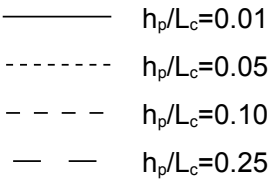

Other models

Sparks, 1997

Collombet, 2009 


\section{a)}

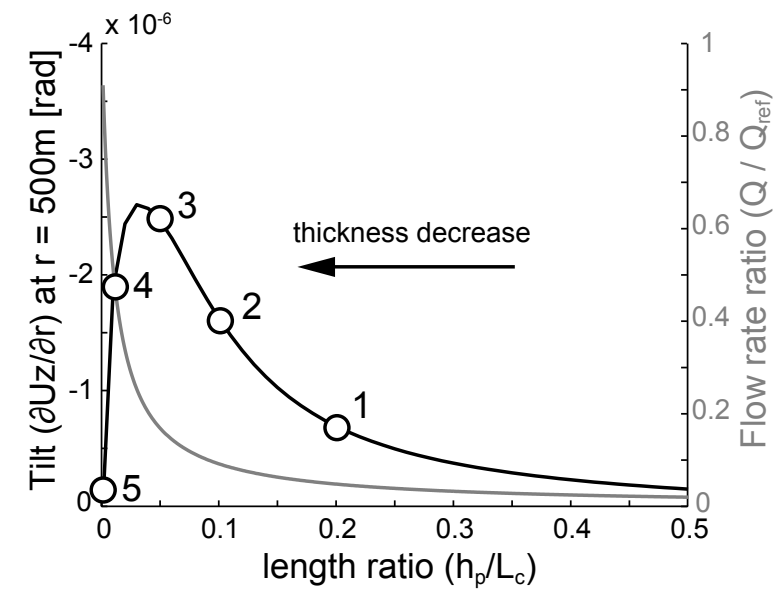

b)

case 1

(reference) case 2

INFLATION

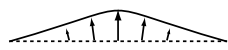

LOW FLUX

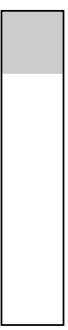

case 3

INFLATION

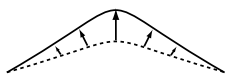

LOW FLUX case 4

SUBSIDENCE

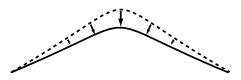

MEDIUM FLUX case 5

SUBSIDENCE

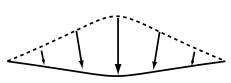

HIGH FLUX
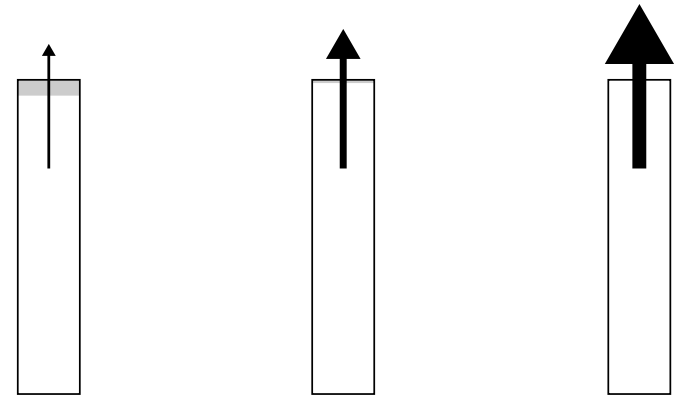
a)
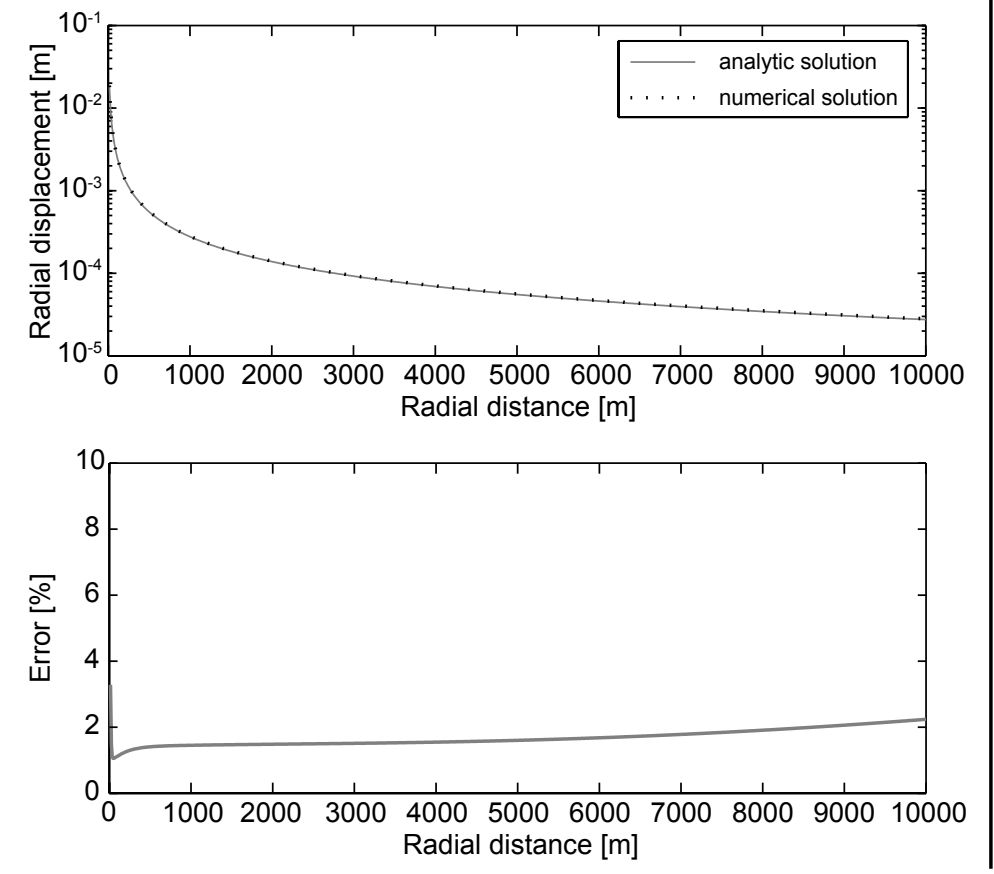

b)
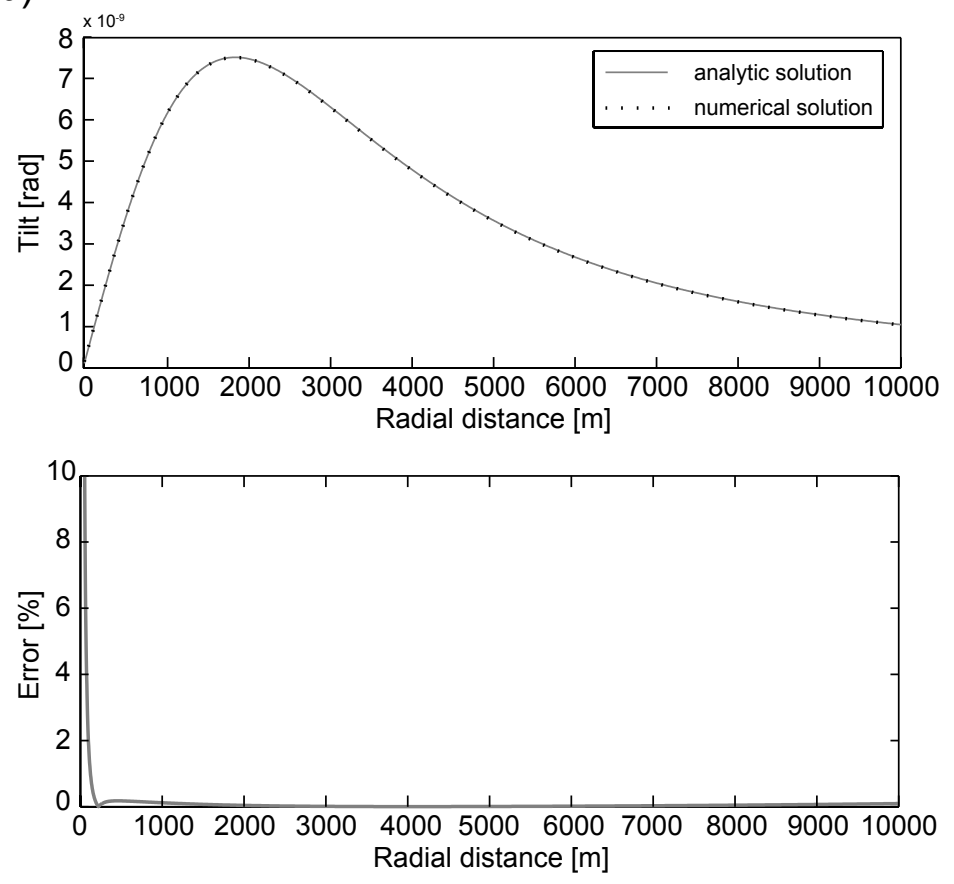